\title{
A Multiresolution Autofocusing Method for Automated Microscopy
}

\author{
Qiang Wu, Xiangyou Li, Tiehan Chen, Yuping Wang and Kenneth R. Castleman
}

Advanced Digital Imaging Research, LLC

2450 South Shore Blvd. Ste.305

League City, TX 77573

Autofocusing is crucially important for automated microscopy, where fully automatic acquisition of microscope images in unattended operation is required. In addition to providing automation of imaging for scanning microscopy applications, it facilitates objective, accurate, and consistent image measurements for quantitative analysis. Autofocusing algorithms determine the in-focus position for an image based on maximization of a focus function, which represents the measure of focus as a function of the axial z position, and is sampled at different positions along the $\mathrm{z}$-axis. The value of the focus function is computed from an image captured at that $\mathrm{z}$ position. Research on microscope autofocusing dates back over a quarter of a century, and many autofocusing functions and algorithms have been proposed in the literature [1-4]. A commonality of existing autofocusing methods is that all of them are based on single-resolution image analysis, and have inherent limitations. Recent developments in signal processing and wavelet transform theory, however, suggest an alternative approach to tackle the problem and to overcome the limitations by resorting to multiresolution image analysis.

This paper presents a multiresolution autofocusing method. In comparison with the single-resolution approach, the new method has the following advantages: (1) Using a wavelet-based multiresolution image representation, focus function computations and subsequently the search for best focus position can be performed flexibly at one or more image resolutions. (2) Since noise in microscope images is manifested mostly at the imaging resolution and ignorable at certain lower resolutions, focus functions computed at a lower resolution tend to be less vulnerable to noise that causes false maximums, and thus making the search for best focus position much easier and more reliable. (3) The ability to utilize lower resolution representations of the image for focus measurement and search can drastically reduce the computation time and therefore leads to substantially faster autofocusing algorithms. Fig. 1 (left) and (right) show respectively an in-focus and an out-of-focus FISH image represented in multiresolution wavelet transform. The differences between the in-focus and out-of-focus images are remarkable even at lower resolution, and can thus be exploited to speedup the focus function computation. Fig. 2 plots the curves of a focus function measured from the image representation of high to low resolution (from A to C). Clearly the lower the resolution the less noisy the focus curve becomes.

The proposed method employs a Haar transform to generate a multiresolution image representation. Then across different resolutions the image power is computed as the focus measure and a coarse-to-fine search is performed to find the best focus position [2]. The method was tested for fluorescence microscopy on 30 fields of FISH cytogenetic specimens (DAPI channel images), along with several current best-performing methods. Experimental results summarized in Table 1 show that the proposed multiresolution technique significantly outperforms those existing single-resolution methods in terms of the focus accuracy, reliability, and speed.

References:

[1] Jarvis, R. A., Microscopy 24:163, 1976. 
[2] F. R. Boddeke et. al., Bioimaging 2:193-203, 1994.

[3] A. Santos et. al., J. Microscopy 188:264-272, 1997.

[4] J. M. Geusebroek et al., Cytometry 39:1-9, 2000
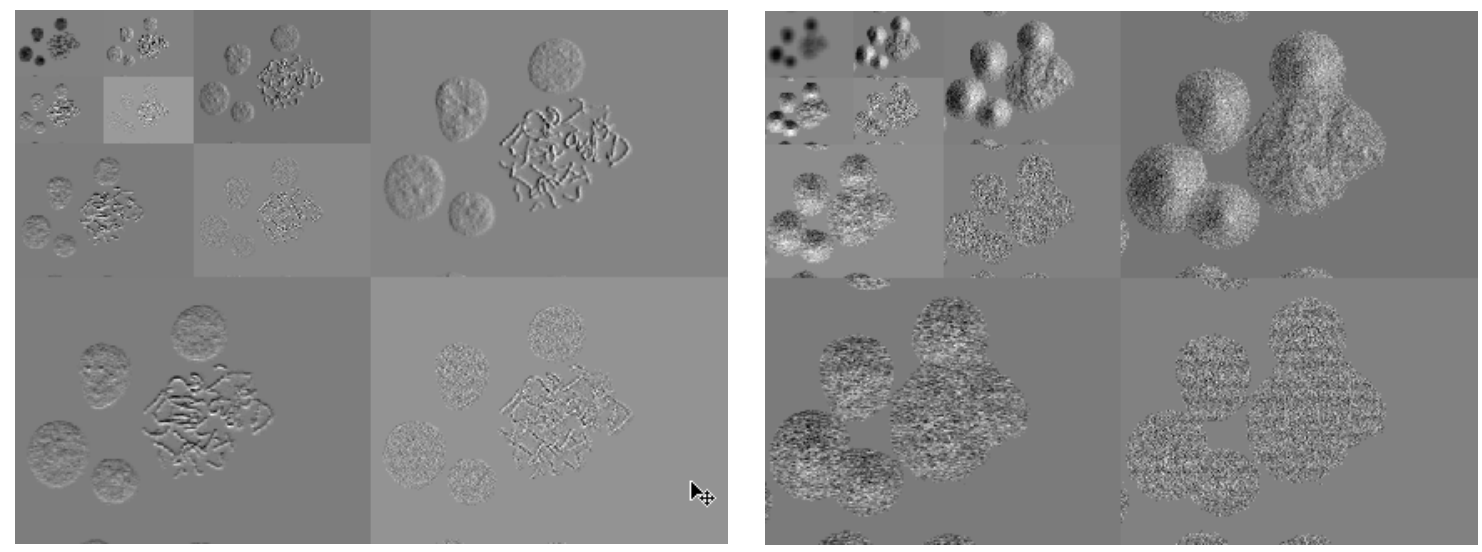

Figure 1. The left and the right images show an in-focus and an out-of-focus FISH image represented in their multiresolution wavelet transform respectively.



Figure 2. The focus function curves measured at three different resolutions.

\begin{tabular}{||c|c|c|c|c|c||}
\hline & $\begin{array}{c}\text { BRENNER } \\
\text { FILTERING }\end{array}$ & $\begin{array}{c}\text { BANDPASS } \\
\text { FILTERING }\end{array}$ & $\begin{array}{c}\text { GAUSSIAN } \\
\text { DERIVATIVE }\end{array}$ & AUTOCORRELATION & $\begin{array}{c}\text { PROPOSED } \\
\text { METHOD }\end{array}$ \\
\hline Focus Error $(\%)$ & 7.6 & 27.77 & 8.2 & 7.4 & 0.13 \\
\hline Error Mean $(\mu \mathrm{m})$ & 0.4215 & 0.6108 & 0.5514 & 0.5001 & 0.0276 \\
\hline Error S.D. $(\mu \mathrm{m})$ & 1.5214 & 2.3222 & 1.9520 & 1.9069 & 0.2880 \\
\hline Time (\# of ticks) & 52.6427 & 46.8550 & 364.3900 & 35.9037 & 17.9220 \\
\hline
\end{tabular}

Table 1. Experimental results of various focus methods averaged over 100 equidistant starting positions and 30 stacks of FISH DAPI channel images. These images were captured at a z-axis step size of $0.05 \mu \mathrm{m}$ over an interval of $30 \mu \mathrm{m}$. Focus error refers to those errors greater than 0.1 $\mu \mathrm{m}$. 